\title{
Factors contributing to ageing of high voltage carbon/carbon supercapacitors in salt aqueous electrolyte
}

\author{
Paula Ratajczak • Krzysztof Jurewicz • \\ François Béguin
}

Received: 12 August 2013/ Accepted: 25 October 2013/Published online: 10 November 2013

(C) The Author(s) 2013. This article is published with open access at Springerlink.com

\begin{abstract}
Accelerated ageing based on floating has been used to investigate the state of health of high voltage carbon/carbon capacitors in aqueous $\mathrm{Li}_{2} \mathrm{SO}_{4}$ electrolyte and to determine the factors influencing their life-time. A pressure sensor connected to the cell allowed to measure gas evolution caused by overcharging of the system and to determine the maximum operating voltage limit of the cell. At voltage higher than $1.5 \mathrm{~V}$, gases started to evolve, together with oxidation of the positive carbon electrode material and formation of decomposition and/or corrosion products. The positive electrode was found to entail the ageing of the entire system, being covered by twice more surface oxygenated groups than the negative one after $120 \mathrm{~h}$ of floating at $1.7 \mathrm{~V}$. The blockage of pore entrances by oxygenated surface functionalities decreases the accessible pore volume, causing a decrease of capacitance and an increase of internal resistance during floating. A drop of capacitive current is observed at voltage higher than $1.2 \mathrm{~V}$ on the voltammograms already after $20 \mathrm{~h}$ floating at $1.7 \mathrm{~V}$. From the study, it can be concluded that the AC/AC supercapacitors using aqueous $\mathrm{Li}_{2} \mathrm{SO}_{4}$ with stainless steel current collectors can operate safely up to $1.5 \mathrm{~V}$, which is actually much more than the voltage value of $0.8-1 \mathrm{~V}$ reached with aqueous $\mathrm{KOH}$ and $\mathrm{H}_{2} \mathrm{SO}_{4}$ electrolytes.
\end{abstract}

Keywords AC/AC supercapacitor - Salt aqueous electrolyte · Ageing · Floating · Pressure evolution . High voltage

P. Ratajczak · K. Jurewicz · F. Béguin $(\bowtie)$

Institute of Chemistry and Technical Electrochemistry, Poznan University of Technology, Piotrowo 3, 60-965 Poznan, Poland e-mail: francois.beguin@ put.poznan.pl

\section{Introduction}

In comparison to organic media, the main drawback of supercapacitors (SCs) using acidic and basic aqueous electrolytes is a much lower electrochemical window. With organic electrolytes, the operating voltage attains $2.7 \mathrm{~V} \mathrm{[1]}$ and only ca. $1.0 \mathrm{~V}$ with $\mathrm{H}_{2} \mathrm{SO}_{4}$ and $\mathrm{KOH}[2,3]$. However, aqueous solutions demonstrate a better conductivity than the organic ones, and from a technological point of view they do not require purification and highly expensive drying processes. Therefore, it is interesting to search for electrolytes which would allow to increasing the operating voltage of SCs while using aqueous solutions. In this context, it has been recently demonstrated that SCs using aqueous alkali sulfate electrolytes are able to operate up to $2 \mathrm{~V}$ under galvanostatic charge/discharge cycling (ca. 10,000-15,000 cycles) $[3,4]$. Due to the low cost and environment friendly character of these systems, it is now necessary to check if they could withstand such voltage after a much higher number of cycles. Since such tests would require extremely long periods of time, it is needed to apply an alternative methodology to prove the lifetime stability of the device.

Taking into account most of the envisioned applications of SCs [5, 6], ageing by keeping the system at an imposed high voltage is an accurate method to examine the state-ofhealth $(\mathrm{SOH})$ of a cell, while not requiring an extremely time consuming test $[7,8]$. The main symptoms during SC ageing are a loss of capacitance, an increase of equivalent series resistance (ESR) and an increase of internal pressure [9]. Therefore, to know perfectly the $\mathrm{SOH}$ of a $\mathrm{SC}$, it is necessary to monitor simultaneously these three parameters at various periods of time during the operation of the system. A SC is usually recognized as out of service when the ESR is increased by $100 \%$ and/or the initial capacitance reduced by $20 \%$ [10]. 
The main objective of this study was to evaluate the $\mathrm{SOH}$ of AC/AC SCs operating in aqueous lithium sulfate electrolyte and to determine their performance limits by accelerated ageing under potentiostatic conditions, called floating. After identifying the possible perturbation phenomena, i.e., evolution of gases, oxidation of carbon, formation of corrosion products at the electrode-current collector interface, it is concluded that the AC/AC SC in aqueous $\mathrm{Li}_{2} \mathrm{SO}_{4}$ can operate with a long cycle life at voltage as high as $1.5 \mathrm{~V}$.

\section{Experimental}

\subsection{Materials and electrolyte}

The electrodes for the study were composed of $85 \mathrm{wt} \%$ active material (activated carbon DLC SUPER 30, NOR$\mathrm{IT}^{\circledR}$ ) further labeled as AC, $10 \mathrm{wt} \%$ polyvinylidene fluoride as binder (PVdF, Kynar HSV900, Arkema) and 5 wt $\%$ carbon black (C65, Timcal). They were prepared in the form of pellets $(1 \mathrm{~cm}$ diameter, thickness ca. $0.3 \mathrm{~mm}$ and mass $8-10 \mathrm{mg}$ ) pressed under $4,870 \mathrm{~kg} \mathrm{~cm}^{-2}$. For thermoprogrammed desorption (TPD) analysis, self-standing electrodes from activated carbon cloth (ACC 507-20, Ky$\left.\mathrm{nol}^{\circledR}\right)$ were selected to avoid the interference of the electrode binder. Glassy microfiber filter (GF/A, Whatman ${ }^{\mathrm{TM}}$ ) of thickness $260 \mu \mathrm{m}$ was used as separator. The electrolyte was $1 \mathrm{~mol} \mathrm{~L}^{-1} \mathrm{Li}_{2} \mathrm{SO}_{4}$ (Sigma Aldrich $\geq 99.0$ ).

\subsection{Accelerated ageing protocol}

The electrochemical investigations were realized with a multichannel VMP3 potentiostat/galvanostat (Biologic Instrument, France) using two-electrode cells assembled in a Teflon Swagelok $^{\circledR}$ vessel with stainless steel $(316 \mathrm{~L}$ grade) current collectors; the separator was sandwiched between two carbon electrodes. During accelerated ageing, 2-h periods of potentiostatic mode (called 'floating') were followed by five galvanostatic $\left(1 \mathrm{~A} \mathrm{~g}^{-1}\right.$ referred to the active mass of one electrode) cycles (Fig. 1), and capacitance and ESR were estimated from the fifth discharge to monitor the $\mathrm{SOH}$ after each floating period. The ESR was determined as the voltage difference measured at current and currentless conditions divided by the applied current (so-called Ohmic drop). The floating and galvanostatic sequences presented in Fig. 1 were repeated 60 times, i.e. for a total floating time of $120 \mathrm{~h}$. In our investigations, the ageing test was realized at maximum voltages of 1.5, 1.6, 1.7 and $1.8 \mathrm{~V}$. The gas evolution was detected with a digital pressure sensor KELLER 35X Ei (pressure range 0-3 bars; total error band of $0.05 \%$ ) connected to the Swagelok cell. We used a very small excess of electrolyte

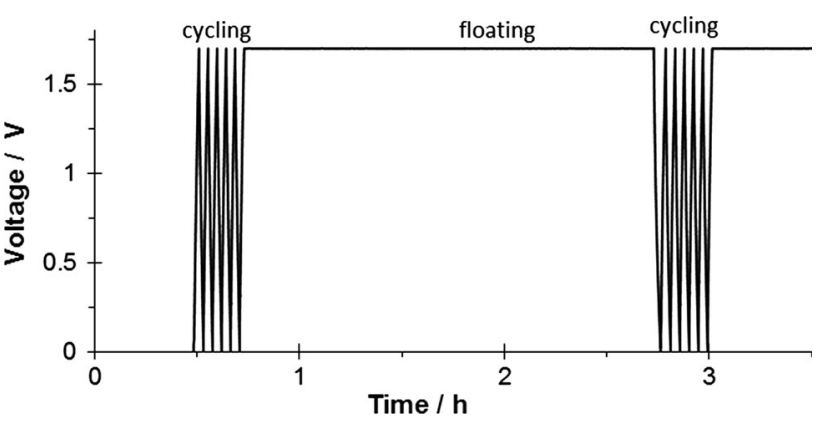

Fig. 1 Schematic representation of the voltage profile during one floating sequence and during the five galvanostatic cycles preceding and following this sequence; the fifth discharge after each floating sequence was considered to estimate the capacitance and ESR values

to minimize the contribution of gas dissolution on the accuracy of pressure measurements, and the system was completely filled, such a way that the dead volume was negligible.

\subsection{Surface functionality analysis}

To investigate the evolution of surface chemistry after accelerated ageing, ACC electrodes were taken out from SCs after $120 \mathrm{~h}$ of floating, washed carefully with distilled water to remove the electrolyte and dried under vacuum at $120{ }^{\circ} \mathrm{C}$. The surface oxygenated functionality of the carbon electrodes was characterized by temperature-programmed desorption (TPD), using a TG equipment (TG209 F1 Iris, NETZSCH) coupled to a mass spectrometer (QMS 403C Aëolos, NETZSCH). In these experiments c.a. $6 \mathrm{mg}$ of ACC sample was heated up to $950{ }^{\circ} \mathrm{C}$ (heating rate $20{ }^{\circ} \mathrm{C} \mathrm{min}^{-1}$ ) under a helium flow rate of $50 \mathrm{~mL} \mathrm{~min}{ }^{-1}$. The quantification of $\mathrm{CO}_{2}$ and $\mathrm{CO}$ groups was done after calcium oxalate monohydrate calibration, taking into account the $\mathrm{CO}$ disproportionation.

\section{Results and discussion}

The $\mathrm{SOH}$ of SCs has been monitored by measuring capacitance, resistance and pressure evolution. However, considering applications of SCs, such as emergency and back-up devices, where the system requires to be kept in charged state for long time, the monitoring of leakage current variations during floating is also very important to understand the ageing mechanism.

The leakage current was recorded during each 2-h potentiostatic (floating) period [11, 12]; the current profile during one potentiostatic sequence at $1.6 \mathrm{~V}$ is shown in Fig. 2a. Once the capacitor is fully charged, the leakage current rapidly decreases within few minutes and it goes to equilibrium within longer time. The phenomenon can be 

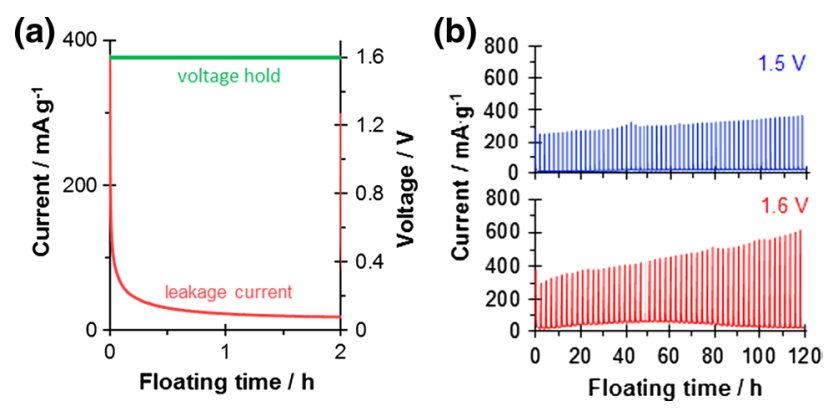

Fig. 2 a Leakage current profile of an AC/AC capacitor in $1 \mathrm{~mol} \mathrm{~L}-1$ $\mathrm{Li}_{2} \mathrm{SO}_{4}$ electrolyte during one 2-h floating period at $1.6 \mathrm{~V}$. b Evolution of leakage current during 60 series at 1.5 and $1.6 \mathrm{~V}$

explained by the double-layer structure with a diffusion layer, where ions have weak interactions with the AC electrode, and a compact layer with ions strongly interacting with the electrode. Initially, the ions of the bulk flow to the diffusion layer resulting in a drastic decay of current. Then the ions of the diffusion layer are pushed to the compact layer, until the structure of the electrical doublelayer is ordered, reaching equilibrium [13]. Figure $2 b$ shows the evolution of leakage current during $120 \mathrm{~h}$ of ageing at 1.5 and $1.6 \mathrm{~V}$. While the profile is almost constant during the repeated floating sequences at $1.5 \mathrm{~V}$, one observes that the initial values of current measured at the beginning of each floating sequence at $1.6 \mathrm{~V}$ increase with the number of floating periods, indicating a slower transition from charging current to leakage current. Furthermore, from the fourth floating sequence at $1.6 \mathrm{~V}$, the equilibrium leakage current increases, reaching its maximum value at ca. 50 cumulated hours. Such increase indicates side reactions, i.e. corrosion, oxidation of carbon or/and gas evolution, for which the current is utilized.

To better understand what is happening with the SC submitted to floating at $1.6 \mathrm{~V}$, it is interesting to compare the evolution of leakage current and capacitance (Fig. 3). Floating at $1.5 \mathrm{~V}$ has small impact on capacitance, which very slowly increases during ageing. By contrast, at $1.6 \mathrm{~V}$, the capacitance rapidly increases during the very first sequences, then increases more slowly up to ca. $50 \mathrm{~h}$ and finally decays rapidly when floating is prolonged. By comparing Figs. 3a and $2 b$, it is interesting to note that both the capacitance and the equilibrium leakage current increase during the first $50 \mathrm{~h}$ of floating at $1.6 \mathrm{~V}$, revealing an improved access of ions to hardly accessible pores of the carbon electrodes under the effect of the electric field. The capacitance decays when ageing of the system dominates. Further, we will show that this capacitance decay after $50 \mathrm{~h}$ of floating at $1.6 \mathrm{~V}$ can be explained by the reduction of accessible surface area of the carbon electrodes; in parallel the equilibrium leakage current decreases because some pores are no longer accessible to the obstruction by oxygenated groups.
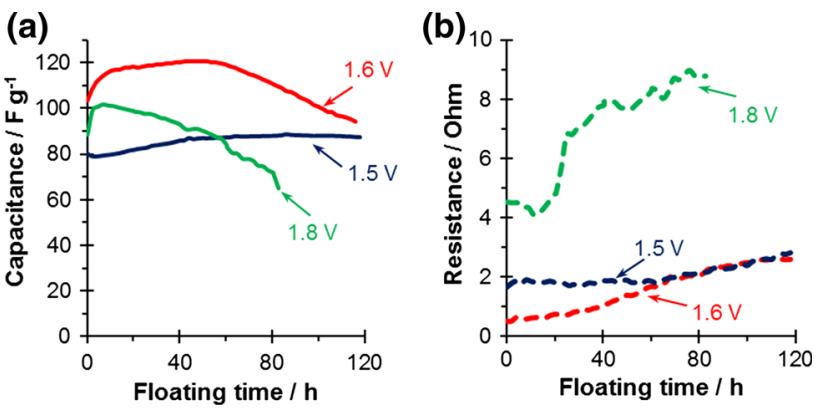

Fig. 3 Effect of floating at different voltage values on a the capacitance and $\mathbf{b}$ ESR of an AC/AC SC in $1 \mathrm{~mol} \mathrm{~L}^{-1} \mathrm{Li}_{2} \mathrm{SO}_{4}$

Considering now the ESR, Fig. $3 b$ shows a stable resistance during floating at $1.5 \mathrm{~V}$, confirming almost no ageing of the AC/AC capacitor at this voltage, while the ESR is stable during the first $30 \mathrm{~h}$ at $1.6 \mathrm{~V}$ floating and then increases continuously for longer floating time. In other words, at $1.6 \mathrm{~V}$, less and less charge is utilized after further floating series, as demonstrated by the lower drop from charging current to leakage current at the beginning of each floating series. According to the end-of-life criteria, while the capacitance dropped by $20 \%$ after $120 \mathrm{~h}$ floating at $1.6 \mathrm{~V}$ (Fig. 3a), the resistance already increased by $100 \%$ after $50 \mathrm{~h}$ only (Fig. 3b). In other words, after $50 \mathrm{~h}$, the system cannot be longer considered as in good $\mathrm{SOH}$.

As the floating voltage increases from 1.5 to $1.6 \mathrm{~V}$ and $1.8 \mathrm{~V}$, the initial capacitance increases more and more rapidly (Fig. 3), meaning that the packing of ions in the smallest pores is better promoted as the electric field increases. However, after a few $2 \mathrm{~h}$ potentiostatic periods at $1.8 \mathrm{~V}$, the capacitance rapidly decays while the ESR increases, indicating that the side reactions are extremely important. Moreover, the very high initial resistance of $4.5 \Omega$ at $1.8 \mathrm{~V}$ suggests some degradation of carbon or/and decomposition of electrolyte during initial test cycles, with possible accumulation of gases at the current collector/ electrode material interface. Therefore, to verify that gases could be at the origin of some troubles, internal pressure evolution has been measured during floating.

The influence of maximum voltage on internal overpressure versus time during few galvanostatic cycles with a current density of $1 \mathrm{~A} \mathrm{~g}^{-1}$ is shown on Fig. 4. As the test time proceeds at $1.8 \mathrm{~V}$, the pressure increases under galvanostatic charging and, as shown in our other work [14], it increases constantly during floating at $1.8 \mathrm{~V}$ to reach 95 mbar after $1 \mathrm{~h}$. It can be seen that even charging/discharging at $1.5 \mathrm{~V}$ can generate some amount of gases. However, the previous capacitance, ESR and leakage current measurements at floating voltage of $1.5 \mathrm{~V}$ demonstrated that such small gas evolution is not detrimental for the $\mathrm{SOH}$ of the AC/AC capacitor. The higher internal pressure evolution at $1.8 \mathrm{~V}$ indicates clearly more 


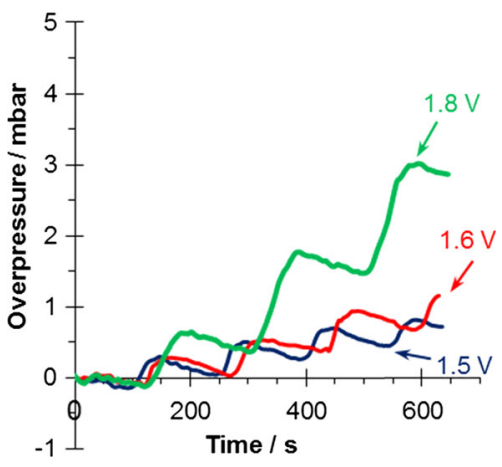

Fig. 4 Cell internal overpressure during galvanostatic cycles with a current density of $1 \mathrm{~A} \mathrm{~g} \mathrm{~g}^{-1}$ up to $1.5,1.6$ and $1.8 \mathrm{~V}$

decomposition of the electrolyte, which can finally lead to electrolyte depletion and/or lower cohesion of the electrode material. As far as gas evolution is concerned, there is not a noticeable difference of pressure evolution between 1.5 and $1.6 \mathrm{~V}$.

Furthermore, it was also observed that for the AC/AC systems under floating voltage higher than $1.6 \mathrm{~V}$, the ageing is also related to corrosion of the stainless steel current collectors, whose products deposit mainly on the positively polarized collector [14]. The corrosion process has important influence on the fading performance of the SC by worsening the electrode-collector contact and blocking a part of pores of AC by decomposition and/or corrosion products. For example, in organic medium, it has been previously shown that the by-products from electrolyte decomposition are deposited on the components of the system and are responsible of simultaneous changes of capacitance and resistance [10, 15].

The cyclic voltammograms (CV) of the fresh cells (Fig. 5; dotted line) display the typical rectangular shape for a SC. By contrast the CVs recorded after each 20 series of floating sequences at 1.5 and $1.7 \mathrm{~V}$ revealed a more resistive character (Fig. 5). When recorded up to the ageing voltage of $1.5 \mathrm{~V}$ (Fig. 5b) and $1.7 \mathrm{~V}$ (Fig. 5d), they show a decrease of capacitive current at voltage higher than $1 \mathrm{~V}$. Since this diminishing of current impedes to take profit from higher voltage, it negatively affects the deliverable energy and power density. According to Pell et al. [16], the capacitance decrease and resistance increase can be due to reduced ion availability, called 'electrolyte starvation effect'. If the electrolyte reservoir is too small compared to the working surface area of the electrodes, the efficiency of SC's operation is reduced as a function of state-of-charge (SOC) [17]. In systems where electrolyte starvation cannot be the cause of CVs narrowing with voltage increase, Mysyk et al. [18] have proved that the current decay is due to the saturation of the active surface area of the electrode material by the stored ions. Therefore, the fading of capacitive current which appears at voltage higher than $1 \mathrm{~V}$
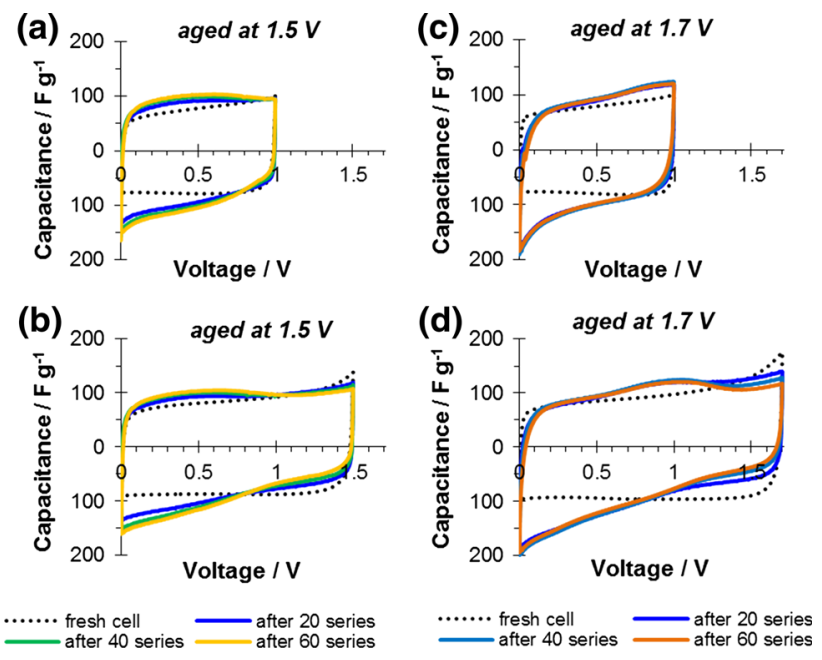

Fig. $5 \mathrm{CV}$ at $10 \mathrm{mVs}^{-1}$ scan rate recorded after each 20 series of 2-h floating sequences at $1.5 \mathrm{~V}(\mathbf{a}, \mathbf{b})$ and $1.7 \mathrm{~V}(\mathbf{c}, \mathbf{d})$ on an $\mathrm{AC} / \mathrm{AC}$ capacitor in $1 \mathrm{~mol} \mathrm{~L}^{-1} \mathrm{Li}_{2} \mathrm{SO}_{4}$

on the CVs after the floating series, while not appearing on the fresh cells (Fig. 5), must be attributed to the reduced accessible surface area of activated carbon for ions, due to the deposition of decomposition/corrosion products in the porosity of the positive electrode. The change of CVs shape just after 20 series demonstrates worse charge propagation which explains an increase of cell resistance related with such products formation. It is also worth to notice, that this issue becomes more significant in further test series. Furthermore, the increase of capacitive current at discharged state for the cells aged at 1.5 and $1.7 \mathrm{~V}$ (Fig. 5a, c) suggests a reversible pseudocapacitive behavior, which could be related with modified surface functionality.

Many properties of activated carbons are strongly influenced by their surface functionality. Therefore, the characterization and quantification of oxygenated surface groups could be helpful for explaining the changes demonstrated in Figs. 2, 3, 5. The increase in oxygen content modifies the electrostatic field on the surface, which makes the micropores of carbon more easily wetted in aqueous solutions [19]. Figure 6 shows the weight loss, $\mathrm{CO}_{2}$ and $\mathrm{CO}$ profiles obtained by TPD for the untreated ACC and electrochemically aged electrodes after 120 cumulated hours of floating at 1.7 V. The important weight loss for the positive electrode, essentially related to $\mathrm{CO}_{2}$ evolution, together with a lesser amount of $\mathrm{CO}$, indicates an important surface oxidation of carbon (Fig. 7). Comparing the TPD patterns, it is clear that the surface functionality of the aged negative electrode is not very different from the pristine ACC, whereas the positive electrode is not only rich with new oxygenated groups, but also some reactions occurred resulting in surface modification (disappearance of $\mathrm{CO}_{2}$ peak at $850{ }^{\circ} \mathrm{C}$ ). The cumulated amount of $\mathrm{CO}_{2}$ evolved is of 3.6 and $2.2 \mathrm{mmol} \mathrm{g}^{-1}$ for the positive and negative aged ACC electrodes, respectively, as 

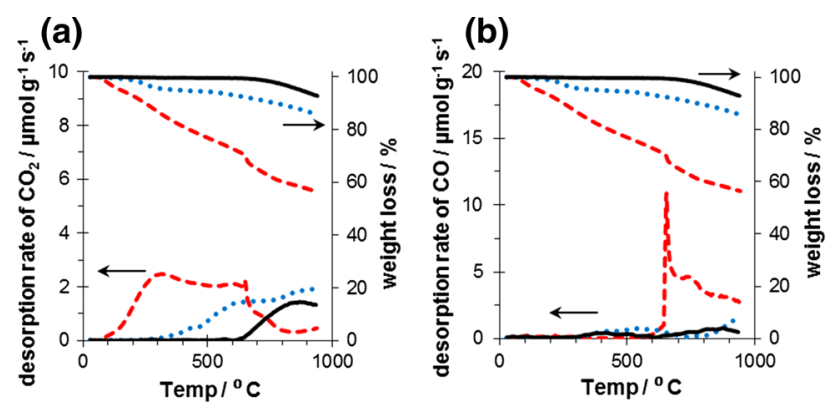

Fig. 6 TPD on the pristine ACC (full line) and on aged positive (dashed line) and negative (dotted line) carbon electrodes after $120 \mathrm{~h}$ of floating at $1.7 \mathrm{~V}$ in $1 \mathrm{molL}^{-1} \mathrm{Li}_{2} \mathrm{SO}_{4}$ : a $\mathrm{CO}_{2}$ evolution; b $\mathrm{CO}$ evolution (a)

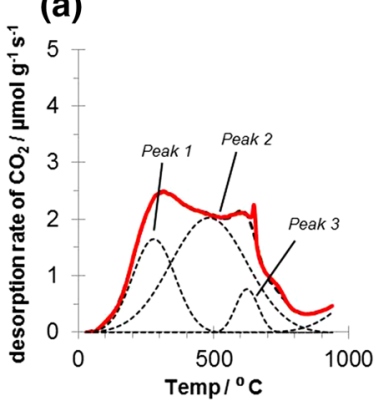

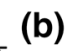

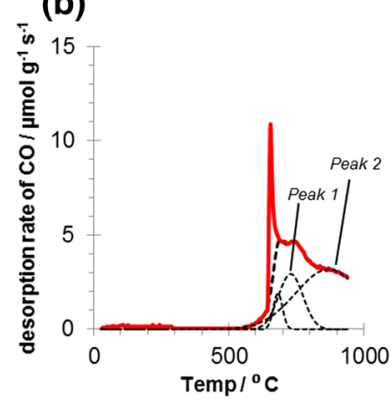

Fig. 7 Deconvolution of TPD patterns for the positive ACC electrode after $120 \mathrm{~h}$ ageing at $1.7 \mathrm{~V}$ : a $\mathrm{CO}_{2}$ pattern; b $\mathrm{CO}$ pattern (full line TPD experimental data; dashed line individual peaks; thick dashed line sum of the individual peaks)

compared to $0.9 \mathrm{mmol} \mathrm{g}^{-1}$ for the fresh ACC. The CO release is $3.9,1.1$ and $0.9 \mathrm{mmol} \mathrm{g}^{-1}$, for the positive electrode, negative one and fresh ACC, respectively.

To determine the types of oxygenated complexes formed on the surface of the aged positive electrode, the $\mathrm{CO}_{2}$ and $\mathrm{CO}$ patterns have been deconvoluted. A multiple Gaussian function was used for fitting each of the TPD patterns [20, 21]. As it is observed on Fig. 7, the accelerated ageing leads to an increase of the $\mathrm{CO}_{2}$ desorption peaks at $270{ }^{\circ} \mathrm{C}$ (peak 1), $500{ }^{\circ} \mathrm{C}$ (peak 2) and $620{ }^{\circ} \mathrm{C}$ (peak 3) assigned to carboxylic and peroxide groups, respectively [22]. The $\mathrm{CO}_{2}$ peaks at higher temperatures might be originated from lactone groups [23]. The CO TPD pattern includes contributions from quite stable oxygenated complexes, assigned as ether carbonyl/quinone groups at $710{ }^{\circ} \mathrm{C}$ (peak 1) and pyrone-type structure at $920{ }^{\circ} \mathrm{C}$ (peak 2) $[22,23]$. The deconvolution of $\mathrm{CO}_{2}$ and $\mathrm{CO}$ evolution includes also a sharp peak noticeable for the positive electrode ca $700{ }^{\circ} \mathrm{C}$ which, together with a discontinuity in the TG curve (Fig. 6), is probably related with the catalytic desorption of oxygenated surface groups, due to the presence of corrosion products from the stainless steel collectors.
The formation of oxygenated complexes on the surface of the electrode material provokes a partial blockage of the pores entrances, explaining the decay of capacitance observed on Fig. 5 after floating. From the foreword, it is obvious that the resistance increase during floating (Fig. 3) might have, at least partly, its origin in the relatively important oxidation of the positive electrode.

\section{Conclusions}

We have demonstrated that floating in potentiostatic conditions is an accurate method to simulate aging during the performance of AC/AC SCs in aqueous lithium sulfate electrolyte and to monitor their $\mathrm{SOH}$. At voltage higher than $1.5 \mathrm{~V}$, gases are generated, and the electrodes are oxidized (essentially the positive one). The decrease of capacitance with floating seems to be related with the decrease of accessible pore volume, due to the blockage of pore entrances by oxygenated surface functionalities. The increase of resistance during floating might be due to the conjunction of few factors: (i) the oxidation of carbon which decreases the carbon conductivity; (ii) the formation of corrosion products at the interface between the current collector and the carbon electrode; (iii) the evolution of gases which worsen the collector/electrode contact but may also occupy partly the porosity of carbon. Notwithstanding all these possible perturbation phenomena, from the presented study, it is now possible to asses that AC/AC SCs in lithium sulfate electrolyte can operate with a very long cycle life at voltage as high as $1.5 \mathrm{~V}$. This value is remarkably high for an aqueous electrolyte compared to the only $0.7-0.8 \mathrm{~V}$ generally possible when $\mathrm{KOH}$ or $\mathrm{H}_{2} \mathrm{SO}_{4}$ are used as electrolyte. On the other hand, the study also clearly demonstrates that galvanostatic cycling with limited number of cycles (ca 10,000) is definitively not an accurate test to determine the maximum operating voltage of SCs. In other words, most of the claims in literature must be considered with great care, meaning that the values of voltage indicated must be reduced by around $0.3-0.4 \mathrm{~V}$, especially when gold current collectors were used.

Acknowledgments The authors are grateful to the Foundation for Polish Science (FNP) for funding the ECOLCAP project realized within the WELCOME program, co-financed from European Union Regional Development Fund. Norit is acknowledged for providing the activated carbon, Arkema for providing the Kynar PVDF binder and Timcal for providing the C65 carbon black.

Open Access This article is distributed under the terms of the Creative Commons Attribution License which permits any use, distribution, and reproduction in any medium, provided the original author(s) and the source are credited. 


\section{References}

1. Burke A (2000) Ultracapacitors: why, how, and where is the technology? J Power Sources 91:37-50

2. Ruiz V, Santamaria R, Granda M, Blanco C (2009) Long-term cycling of carbon-based supercapacitors in aqueous media. Electrochim Acta 54:4481-4486

3. Demarconnay L, Raymundo-Piñero E, Béguin F (2010) A symmetric carbon/carbon supercapacitor operating at $1.6 \mathrm{~V}$ by using a neutral aqueous solution. Electrochem Commun 12:1275-1278

4. Fic K, Lota G, Meller M, Frąckowiak E (2012) Novel insight into neutral medium as electrolyte for high-voltage supercapacitors. Energy Environ Sci 5:5842-5850

5. Shimizu T, Underwood C (2013) Super-capacitor energy storage for micro-satellites: feasibility and potential mission applications. Acta Astron 85:138-154

6. Conte M (2010) Supercapacitors technical requirements for new applications. Fuel Cells 10:806-818

7. Ruch PW, Cericola D, Foelske-Schmitz A, Kötz R, Wokaun A (2010) Aging of electrochemical double layer capacitors with acetonitrile-based electrolyte at elevated voltages. Electrochim Acta 55:4412-4420

8. Weingarth D, Foelske-Schmitz A, Kötz R (2013) A reliable determination method of stability limits for electrochemical double layer capacitors. J Power Sources 225:84-88

9. Zhu M, Weber CJ, Yang Y, Konuma M, Starke U, Kern K, Bittner AM (2008) Chemical and electrochemical ageing of carbon materials used in supercapacitor electrodes. Carbon 46:1829-1840

10. Kötz R, Ruch PW, Cericola D (2010) Aging and failure mode of electrochemical double layer capacitors during accelerated constant load tests. J Power Sources 195:923-928

11. Conway BE (1999) Electrochemical supercapacitors: scientific fundamentals and technological applications. Kluwer, New York

12. Drew J (2007) Supercapacitors can replace a backup battery for power ride-through applications. Design Notes 450 09/08/450
13. Zhou S-Y, Li X-H, Wang Z-X, Guo H, Peng W-J (2007) Effect of activated carbon and electrolyte on properties of supercapacitor. Trans Nonferrous Met Soc China 17:1328-1333

14. Ratajczak P, Jurewicz K, Abbas Q, Béguin F (2013) Electrochim Acta (submitted)

15. Azais P, Duclaux L, Florian P, Massiot D, Lillo-Rodenas MA, Linares-Solano A, Peres JP, Jehoulet C, Béguin F (2007) Causes of supercapacitors ageing in organic electrolyte. J Power Sources 171:1046-1053

16. Pell WG, Conway BE, Marincic N (2000) Analysis of non-uniform charge:discharge and rate effects in porous carbon capacitors containing sub-optimal electrolyte concentrations. J Electroanal Chem 491:9-21

17. Pell WG, Conway BE (2001) Voltammetry at a de Levie brush electrode as a model for electrochemical supercapacitor behaviour. J Electroanal Chem 500:121-133

18. Mysyk R, Raymundo-Piñero E, Béguin F (2009) Saturation of subnanometer pores in an electric double-layer capacitor. Electrochem Commun 11:554-556

19. Bleda-Martinez MJ, Lozano-Castello D, Morallon E, CazorlaAmoros D, Linares-Solano A (2006) Chemical and electrochemical characterization of porous carbon materials. Carbon 44:2642-2651

20. Figueiredo JL, Pereira MFR, Freitas MMA, Orfao JJM (1999) Modification of the surface chemistry of activated carbons. Carbon 37:1379-1389

21. Figueiredo JL, Pereira MFR, Freitas MMA, Orfao JJM (2007) Characterization of active sites on carbon catalysts. Ind Eng Chem Res 46:4110-4115

22. Zielke U, Huttinger KJ, Hoffman WP (1996) Surface-oxidized carbon fibers: I. Surface structure and chemistry. Carbon 34:983-998

23. Zhou JH, Sui ZJ, Zhu J, Li P, Chen D, Dai YC, Yuan WK (2007) Characterization of surface oxygen complexes on carbon nanofibers by TPD, XPS and FT-IR. Carbon 45:785-796 